\title{
Overexpression of astroglial major histocompatibility complex class I in the medial prefrontal cortex impairs visual discrimination learning in mice
}

\author{
Bolati Wulaer ${ }^{1,2 \dagger}$, Kazuhiro Hada ${ }^{1 \dagger}$, Akira Sobue ${ }^{1}$, Norimichi Itoh ${ }^{1}$, Toshitaka Nabeshima ${ }^{2}$, Taku Nagai ${ }^{1}$
} and Kiyofumi Yamada ${ }^{1 *}$

\begin{abstract}
Background: Immune molecules, such as cytokines, complement, and major histocompatibility complex (MHC) proteins, in the central nervous system are often associated with neuropsychiatric disorders. Neuronal MHC class I $(\mathrm{MHCl})$, such as $\mathrm{H}-2 \mathrm{D}$, regulate neurite outgrowth, the establishment and function of cortical connections, and activity-dependent refinement in mice. We previously established mice expressing $\mathrm{MHCl}$ specifically in astrocytes of the media prefrontal cortex (mPFC) using the adeno-associated virus (AAV) vector under the control of the GfaABC1D promoter. Mice expressing the soluble form of $\mathrm{H}-2 \mathrm{D}(\mathrm{sH}-2 \mathrm{D})$ in the $\mathrm{mPFC}(\mathrm{sH}-2 \mathrm{D}$-expressing mice) showed abnormal behaviors, including social interaction deficits and cognitive dysfunctions. However, the pathophysiological significance of astroglial $\mathrm{MHCl}$ on higher brain functions, such as learning, memory, and behavioral flexibility, remains unclear. Therefore, cognitive function in mice expressing $\mathrm{sH}-2 \mathrm{D}$ in astrocytes of the mPFC was tested using the visual discrimination (VD) task.
\end{abstract}

Methods: $\mathrm{sH}-2 \mathrm{D}$-expressing mice were subjected to the VD and reversal learning tasks, and morphological analysis.

Results: In the pretraining, sH-2D-expressing mice required significantly more trials to reach the learning criterion than control mice. The total number of sessions, trials, normal trials, and correction trials to reach the VD criterion were also significantly higher in $\mathrm{sH}-2 \mathrm{D}$-expressing mice than in control mice. A morphological study showed that dendritic complexity and spine density were significantly reduced in the dorsal striatum of sH-2D-expressing mice.

Conclusion: Collectively, the present results suggest that the overexpression of astroglial $\mathrm{MHCl}$ in the mPFC results in impaired VD learning, which may be accompanied by decreased dendritic complexity in the dorsal striatum and mPFC.

Keywords: $\mathrm{MHCl}$, Astrocyte, Touchscreen, Visual discrimination, Reversal learning, Learning and memory, Cognition, Recognition memory

*Correspondence: kyamada@med.nagoya-u.ac.jp

†Bolati Wulaer and Kazuhiro Hada are co-first authors

1 Department of Neuropsychopharmacology and Hospital Pharmacy,

Nagoya University Graduate School of Medicine, 65 Tsurumai-cho,

Showa-ku, Nagoya 466-8560, Japan

Full list of author information is available at the end of the article

\section{Introduction}

The brain is considered to be 'immuno-privileged' because of the lack of classical immune molecules in the central nervous system (CNS) [1, 2]. However, current research suggests the presence of communication between the immune and nervous system for brain functions after the discovery of immune molecules, original author(s) and the source, provide a link to the Creative Commons licence, and indicate if changes were made. The images or other third party material in this article are included in the article's Creative Commons licence, unless indicated otherwise in a credit line to the material. If material is not included in the article's Creative Commons licence and your intended use is not permitted by statutory regulation or exceeds the permitted use, you will need to obtain permission directly from the copyright holder. To view a copy of this licence, visit http://creativecommons.org/licenses/by/4.0/. The Creative Commons Public Domain Dedication waiver (http://creativeco mmons.org/publicdomain/zero/1.0/) applies to the data made available in this article, unless otherwise stated in a credit line to the data. 
such as cytokines, complement, and major histocompatibility complex (MHC) proteins, in the developing and adult brain [3-5]. Among these immune molecules, recent studies highlighted the roles of MHC class I (MHCI) in the brain. MHCI molecules contain a heavy chain and $\beta 2$-microglobulin light chain $[6,7]$. In the immune system, MHCI presents a short polypeptide of 8-10 amino acids from a cytosolic antigen when a cell is infected with a virus. In the CNS, neuronal MHCI molecules regulate neurite outgrowth, cortical connections, activity-dependent refinement in the visual system, and synaptic plasticity $[3-5,8-10]$. Glial MHCI molecules are weakly expressed in normal and healthy brains but are up-regulated under pathological conditions, such as viral infection. A systemic immune stimulation in rodents has been shown to activate astrocytes and microglia in the brain $[11,12]$ and induce MHCI gene expression in non-neuronal cells [13] and MHCII in microglia [14].

To clarify the pathophysiological role of MHCI expression in astrocytes, we previously established mice expressing MHCI specifically in astrocytes of the medial prefrontal cortex (mPFC) using the AAV vector under the control of the GfaABC1D promoter [15]. Mice expressing the soluble form of $\mathrm{H}-2 \mathrm{D}$ (sH-2D) in the mPFC (sH-2D-expressing mice) showed brain dysfunction manifested by impaired social interactions and object recognition memory, which was accompanied by neuropathological changes, including the activation of microglial cells, decreases in parvalbumin-positive cell numbers, and reductions in dendritic spine density in the mPFC. A treatment with GW4869, an inhibitor of exosome synthesis, ameliorated these behavioral and neuropathological changes in $\mathrm{sH}-2 \mathrm{D}$-expressing mice, suggesting that the overexpression of MHCI in astrocytes affects microglial proliferation as well as neuronal numbers and spine densities, thereby leading to social and cognitive deficits in mice, possibly via exosomes produced by astrocytes [15].

In the present study, we investigated higher brain functions, such as learning, memory, and behavioral flexibility, in sH-2D-expressing mice using the visual discrimination (VD) task. It should be noted that the validation of the animal model is shown in our previous report [15]. The touchscreen-based VD task provides high translational validity to further evaluate neuronal projections for higher-order brain functions in mice [1620]. Previous studies indicated that the dorsal striatum is important for VD learning $[18,21]$. Instrumental action and outcome behaviors are known to depend on the dorsal striatum and its connections with the $\operatorname{mPFC}[22,23]$. Therefore, we examined the dendritic and spine morphology of medium spiny neurons in $\mathrm{mPFC}$ projection terminals, the dorsomedial striatum (DMS), and dorsolateral striatum (DLS) [24].

\section{Methods \\ Animals}

C57BL/6 mice (Japan SLC, Shizuoka, Japan) were housed in groups of 4 per cage and maintained under a standard specific pathogen-free environment with a standard 12-h light/dark cycle (lights on at 9:00) at a constant temperature of $23 \pm 1^{\circ} \mathrm{C}$. Animals were given a 1 -week acclimatization period prior to the start of the experiments. They were allowed free access to food and water before the initiation of pretraining for the VD and reversal learning tasks. Only male mice were used in the present study to avoid potential estrus cycle-related performance variability in females [25]. Three separate batches of mice were used in the present study (a total of 53 mice). The sample size for each experiment was determined based on our previous studies with the relevant type of experiment [18, 26]. They were randomly subjected to control or sH-2D viral injection groups as follows: VD and reversal learning (control 4 mice, sH-2D-expressing 4 mice; 2 mice were excluded from the analyses because of natural death [18]); Golgi staining (control 4 mice, sH-2D-expressing 4 mice; [26]). Animals were handled in accordance with the guidelines established by the Institutional Animal Care and Use Committee of Nagoya University, the Guiding Principles for the Care and Use of Laboratory Animals approved by the Japanese Pharmacological Society, and the National Institutes of Health Guide for the Care and Use of Laboratory Animals.

\section{Plasmid and AAV production}

The validation of the animal model is shown in our previous report [15]. To specifically target astroglial MHCI in the mPFC, we used pAAV-2/5 (Cell BioLabs Inc.) under the control of the GfaABC1D promotor. The MHCI/GFP was expressed in glial fibrillary acidic protein (GFAP)-positive astrocytes, but not in neurons or microglia. The transfection efficiencies to GFP+ cells/ GFAP + cells in our experimental conditions were above $70 \%$ in each group [15]. We produced the plasmid and pAAV-2/5 as described previously [15]. Briefly, cDNA for mouse MHCI was amplified by polymerase chain reaction (PCR) from a mouse brain cDNA library using specific primers (sH-2D forward primer, ATGAATTCG CCGCCATGGGGGCGATGGC; sH-2D reverse primer, ATGTCGACCCATCTCAGGGTGAGGGGCT), and inserted into a pCRII-blunt TOPO vector (Invitrogen, Carlsbad, CA, USA). cDNA was subcloned into the EcoRI site of the expression vector pCAGGS-HA, which was a gift from Dr. Kozo Kaibuchi. In the AAV vector, pZac2.1 gfaABC1D-EGFP-P2A-sH-2D was generated 
by replacing EGFP-P2A-sH-2D in tdTomato in pZac2.1gfaABC1D-tdTomato, which was donated by Dr. Baljit Khakh (Addgene plasmid \# 44332). AAV vectors were prepared as described previously [15, 27]. Briefly, plasmids for the AAV vector, pHelper (Cell BioLabs Inc., San Diego, CA, USA), and pAAV-2/5 were transfected into HEK293 cells (Cell BioLabs, Inc.) using Lipofectamine 2000 (Invitrogen). After a 3-day incubation, cells were collected and lysed by freeze and thaw cycles. Cell lysates were incubated with benzonase nuclease (Millipore, Darmstadt, Germany). Cell debris was removed by centrifugation at $10,000 \times g$ at room temperature for $10 \mathrm{~min}$. Supernatants were used as the primary virus. AAV titers were estimated via a quantitative polymerase chain reaction.

\section{sH-2D-expressing mouse model}

We established the sH-2D-expressing mouse model as described previously (Sobue et al. 2018). Briefly, sevenweek-old male mice were anesthetized with tribromoethanol $(250 \mathrm{mg} / \mathrm{kg}$, i.p.) and positioned in a stereotaxic frame (David Kopf, Tujunga, CA, USA). AAV gfaABC1DEGFP-P2A-sH-2D $\left(1 \times 10^{12}\right.$ genome copies $\left./ \mathrm{ml}\right)$ was bilaterally injected into the $\mathrm{mPFC}(+1.5 \mathrm{~mm}$ anteroposterior, $\pm 0.5 \mathrm{~mm}$ mediolateral from the bregma, $-2.5 \mathrm{~mm}$ dorsoventral from the skull) in a volume of $0.5 \mu \mathrm{l} / \mathrm{site}$, according to the mouse brain atlas [28]. AAV gfaABC1DEGFP-P2A was injected as a control group. Three weeks after injections, animals were subjected to behavioral or morphological analyses.

\section{Touchscreen-based VD and reversal learning tasks}

Tasks were performed using the touchscreen chamber system (Phenosys, Berlin, Germany; Brainscience Idea, Osaka, Japan). The experiments were conducted during the light phase each day (13:00-16:00). The protocol used was described in detail in previous studies [18, 29]. Briefly, access to food and water was restricted for $2 \mathrm{~h}$ (17:00-19:00) each day at least 1 week before pretraining in order to provide sufficient motivation to perform the tasks, and food and water restrictions were continued until the end of the task. The body weight was maintained at $85-90 \%$ of non-restricted mice. The task started with 5 -stage pretraining to shape screen-touch behavior in mice (Fig. 1a). In stage 1 , mice were habituated to the touchscreen chamber. They were allowed to freely explore the chamber and rewards were available during the 20 -min session. The criterion was to receive 30 rewards $(20 \mu \mathrm{l}$ of milk) on 2 consecutive days. During stage 2, one window of the touchscreen was illuminated with a white plain square for $30 \mathrm{~s}$ (Fig. 1b). When the stimulus was offset, the reward nozzle came into the chamber and the reward was delivered. The retrieval of milk initiated an inter-trial interval (ITI) of $20 \mathrm{~s}$ before the next image presentation. When the mouse touched the response window during a white plain square presentation, a reward was delivered to accompany the image stimulus termination. Stage 3 proceeded in the same manner as stage 2, except that the mouse was required to touch the response window displaying the image before reward delivery. Each image was displayed until mice touched the response window. The criterion was to receive 30 rewards in a 60 -min session at least once. In addition to the stage 3 procedure, mice had to initiate each trial by approaching the nozzle in stage 4 (Fig. 1c). When the trial started, the nozzle was presented in the operant chamber without a reward. Touching the nozzle resulted in the presentation of an image on the touchscreen. The criterion was the same as that for stage 3 . In stage 5 , mice were introduced to incorrect responses. Mice were punished for touching a blank response window with a 5-s time-out. ITI began after the time-out, and then the next trial was initiated. The criterion in this stage was to complete 30 rewards showing $\geq 75 \%$ accuracy in a 30 -min session on 2 consecutive days, and mice were then moved to the VD task. To prevent location bias, the stimulus was pseudorandomly presented during all training stages; it never showed more than 3 times on the same side in a row. After mice learned how to operate the touchscreen ( $>75 \%$ on 2 consecutive days), they were subjected to the VD task. In the VD task, trial initiation was triggered by mice touching the nozzle, and 2 stimuli (marble and fan) were then presented simultaneously in the 2 response windows (Fig. 1d). One of the stimuli was associated with a reward, while the other was not. Stimuli were presented pseudorandomly and not displayed in the same location for more than 3 trials in a row (excluding correction trials). Stimulus contingencies were counterbalanced. Touching the correct response resulted in the delivery of a reward ( $20 \mu \mathrm{l}$ of milk). When the incorrect response was touched, the stimuli offset immediately and a 5-s time-out period was started. After ITI (20 s), a correction trial was given instead of a new trial. In the correction trial, the same stimulus set was repeatedly presented in the same location until the mouse made a correct response. The criterion of the task was a more than $80 \%$ correct response on 2 consecutive days. The session finished after $60 \mathrm{~min}$ or the completion of 30 trials, whichever comes first. The total numbers of trials, correction trials, and correction errors as well as the percentage of correct responses and the perseveration index (the number of correction trials as a ratio of errors) in different training stages were analyzed. The 


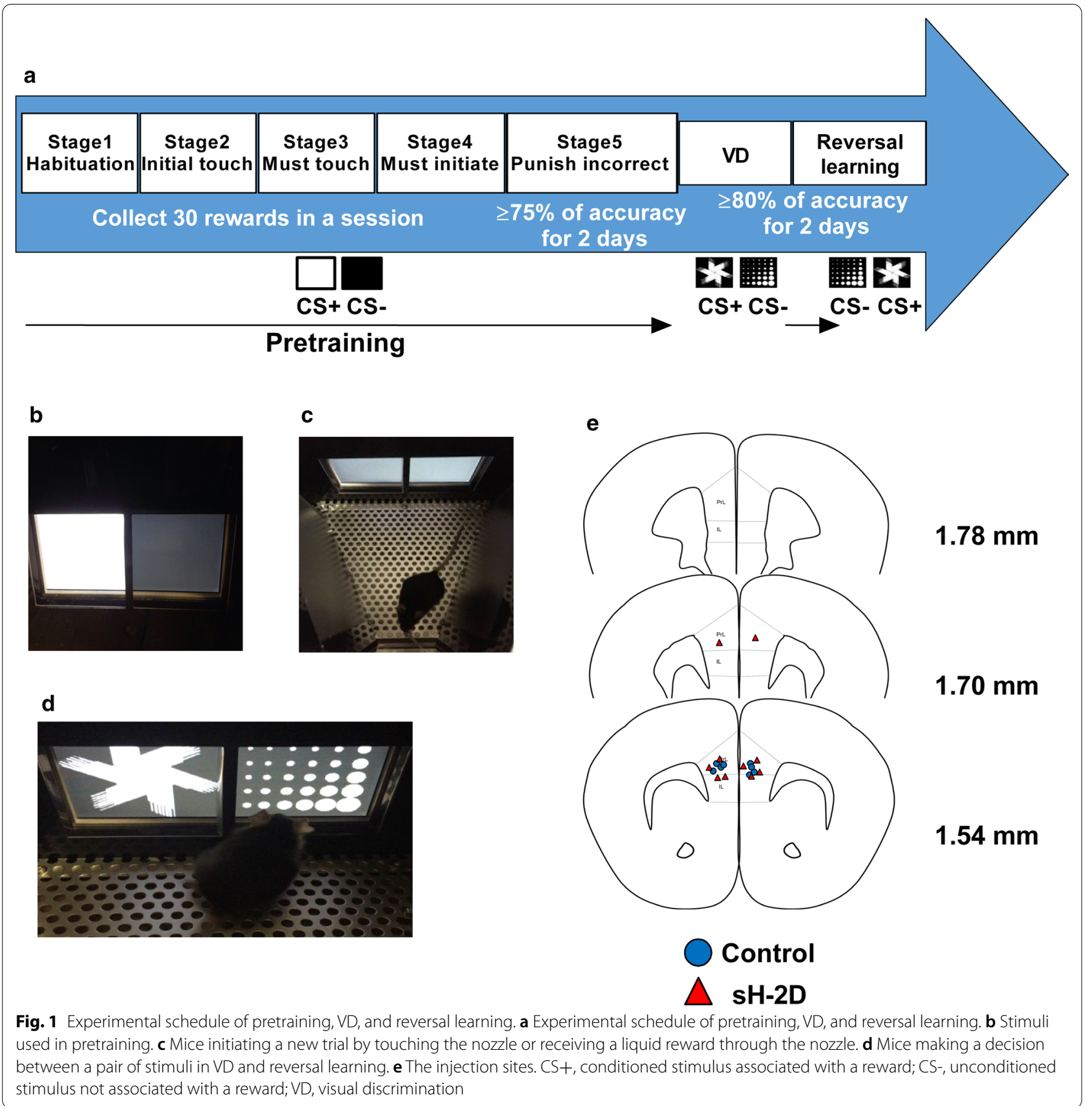

reversal learning task was similar to the initial acquisition of the VD task, except that the contingency of the stimulus pair was reversed. Once a mouse reached the criterion, the contingency of the stimuli was reversed. The previous reward stimulus became an incorrect response, while the previous non-rewarded stimulus became the correct response. The injection sites were checked by immunostaining after the behavioral tasks were completed (Fig. 1e).
Golgi staining and morphological analyses

Golgi staining was performed using the FD Rapid Golgi Stain Kit according to the manufacturer's protocol (FD NeuroTechnologies, Ellicott City, MD, USA) and a previous study [15]. Mice were sacrificed 3 weeks after the viral injection without any stimuli. Brains were then sectioned using a cryostat at a thickness of $80 \mu \mathrm{m}$. Brightfield microscopic images of neurons located in DMS and DLS were obtained (BZ9000, KEYENCE, Osaka, Japan). 
Starting from the soma, circles $10 \mu \mathrm{m}$ apart from each other overlay the dendritic tracings. The length of the dendrites is determined by the number of circles that the dendrites cross over. The intersection is defined as when a branch splits into two sub-branches. Node is the bifurcations on the dendrites and ending is recognized as dendritic endings (terminal ends). Only fully impregnated neurons isolated from neighboring displaying dendritic trees without obvious truncations and impregnated neurons were retained for analyses. We analyzed the secondary or third dendrites on branches of DMS or DLS neurons that were at least $50 \mu \mathrm{m}$ from the cell body. We measured the spines of 3 dendrites per neuron in 3 neurons per mouse. Structural characteristics of spines are shown in Fig. 5d [27]. All images were traced using Neurolucida software (MicroBrightField Bioscience, Williston, VT, USA) and analyzed by NeuroExplorer (MicroBrightField). These analyses were performed using 12 slices per mouse from 4 mice in each group.

\section{Data analyses}

All data were expressed as means \pm SEM. Statistical analyses were performed with GraphPad Prism 6.0 (GraphPad Software, Inc., CA, USA). Differences between two groups were analyzed by a two-tailed Student's t test. Multiple group comparisons were conducted using the analysis of variance, followed by Tukey's test. The criterion for a significant difference was ** $\mathrm{p}<0.01$ or * $\mathrm{p}<0.05$ for all statistical evaluations.

\section{Results}

\section{Performance of $\mathrm{sH}-2 \mathrm{D}$-expressing mice in pretraining} and VD task

Mice were initially subjected to a 5-stages pretraining to gradually shape screen-touching behavior [18, 29]. Pretraining consisted of 5 stages (Fig. 1a). White plain and blank stimuli were used in the pretraining stages (Fig. 1b). In the pretraining, sH-2D-expressing mice required significantly more trials to reach the criterion (to reach $75 \%$ accuracy for at least 2 sessions) than control mice $(\mathrm{t}(6)=2.74, \mathrm{P}=0.0338$; Fig. $2 \mathrm{~b})$. Accordingly, sH-2D-expressing mice appear to have normal visuospatial and motor functions, but impaired reward-associated discriminative learning. The VD task was initiated when mice reached the criterion in pretraining. In this task, mice were required to touch a stimulus to obtain the liquid reward from a pair of stimuli (marble and fan; Fig. 1d, 2c). The VD learning was significantly slower in $\mathrm{sH}-2 \mathrm{D}$-expressing mice than control mice $(\mathrm{P}=0.0414$; Fig. 2d). In fact, $\mathrm{sH}-2 \mathrm{D}$-expressing mice needed more sessions $(\mathrm{t}(6)=2.64, \mathrm{P}=0.0386$; Fig. $2 \mathrm{e})$, trials $(\mathrm{t}(6)=3.35$, $\mathrm{P}=0.0154$; Fig. 2f), normal trials $(\mathrm{t}(6)=2.64, \mathrm{P}=0.0386$; Fig. $2 \mathrm{~g})$, and correction trials $(\mathrm{t}(6)=3.58, \mathrm{P}=0.0116$;
Fig. $2 \mathrm{~h}$ ) to reach the learning criteria (more than $80 \%$ accuracy on 2 consecutive days) than did control mice. Taken together, these results indicate that reward learning was significantly impaired by the overexpression of $\mathrm{MHCI}$ in astrocytes of the mPFC.

\section{Performance of $\mathrm{sH}-2 \mathrm{D}$-expressing mice in reversal learning tasks}

In order to analyze the behavioral flexibility in sH-2D-expressing mice, the animals were then subjected to reversal learning in which the previously incorrect stimulus becomes the correct stimulus and vice versa (Fig. 3a). In a 9-day reversal learning task, the total trials $(\mathrm{t}(6)=0.49$, $\mathrm{P}=0.6379$; Fig. 3b), total normal trials $(\mathrm{t}(6)=0.47$, $\mathrm{P}=0.6427$; Fig. 3c) and total correction trials $(\mathrm{t}(6)=0.09$, $\mathrm{P}=0.9291$; Fig. 3d), were comparable between the groups. No significant differences were observed in the percentage of correct responses (group, $\mathrm{F}(1$, $3)=0.24, \mathrm{P}=0.6565$; sessions, $\mathrm{F}(8,24)=38.88, \mathrm{P}<0.01$; group $\times$ sessions, $\mathrm{F}(8,24)=1.80, \mathrm{P}=0.1272$; Fig. $3 \mathrm{e})$ or the perseveration index (group, $\mathrm{F}(1,3)=0.7183, \mathrm{P}=0.71$; sessions, $F(8,24)=9.14, \mathrm{P}<0.0001$; group $\times$ sessions, $F(8$, $24)=0.40, P=0.9096$; Fig. 3f) between the two groups of mice. Accordingly, the normal capability for reversal learning in $\mathrm{sH}-2 \mathrm{D}$-expressing mice indicates that behavioral flexibility was minimally affected by the overexpression of astroglial MHCI in the mPFC.

\section{Morphology of DMS and DLS neurons in SH-2D-expressing mice}

The dorsal striatum is anatomically divided into DMS and DLS. These areas are involved in VD learning $[18,21]$. These striatal regions receive excitatory inputs from two major sources, the cortex and thalamus, which control contextual, motor, and perceptual decisions [24, 30]. Therefore, we analyzed the morphology of DMS and DLS neurons in control and $\mathrm{sH}-2 \mathrm{D}$-expressing mice by the Sholl analysis (Fig. 4a, f). The dendrites of DMS neurons in sH-2D-expressing mice showed significantly fewer intersections (group, $\mathrm{F}(1,22)=27.79, \mathrm{P}<0.0001$; intersection, $\mathrm{F}(5$, $110)=14.04, \mathrm{P}<0.0001$; group $\times$ intersection interaction, $\mathrm{F}(5,110)=1.173, \mathrm{P}=0.3271$; Fig. $4 \mathrm{~b})$, shorter lengths (group, $\mathrm{F}(1,22)=28.70, \mathrm{P}<0.0001$; length, $\mathrm{F}(5, \quad 110)=17.53, \quad \mathrm{P}<0.0001 ; \quad$ group $\times$ length, $\mathrm{F}(5$, $110)=1.870, \mathrm{P}=0.1053$; Fig. $4 \mathrm{c}$ ), and less nodes (group, $\mathrm{F}(1,22)=28.39, \mathrm{P}<0.0001$; node, $\mathrm{F}(5,110)=7.018$, $\mathrm{P}<0.0001$; group $\times$ node, $\mathrm{F}(5,110)=1.746, \mathrm{P}=0.1301$; Fig. 4d) and endings (group, $F(1,22)=26.82, P<0.0001$; ending, $\mathrm{F}(5,110)=10.60, \mathrm{P}<0.0001$; group $\times$ ending, $F(5,110)=1.23 ; P=0.3000$; Fig. 4e) per Sholl segment, particularly those $10-60 \mu \mathrm{m}$ from the soma, than those in control mice. Similarly, the dendrites of DLS neurons 


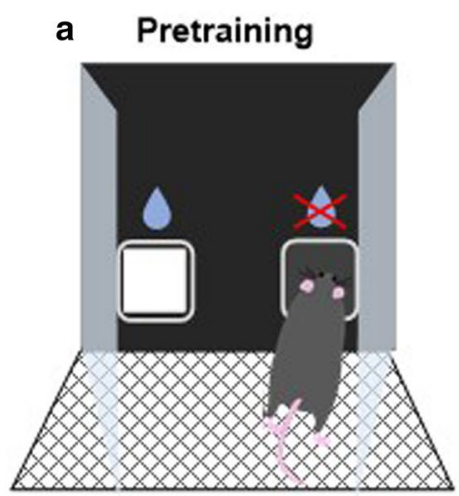

c Visual discrimination task

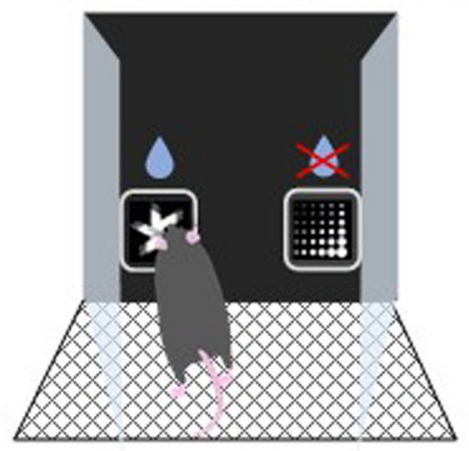

f

Total trials

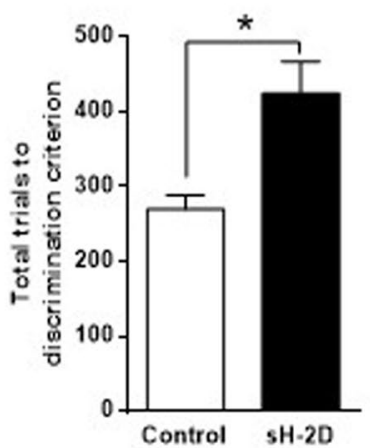

b

Pretraining
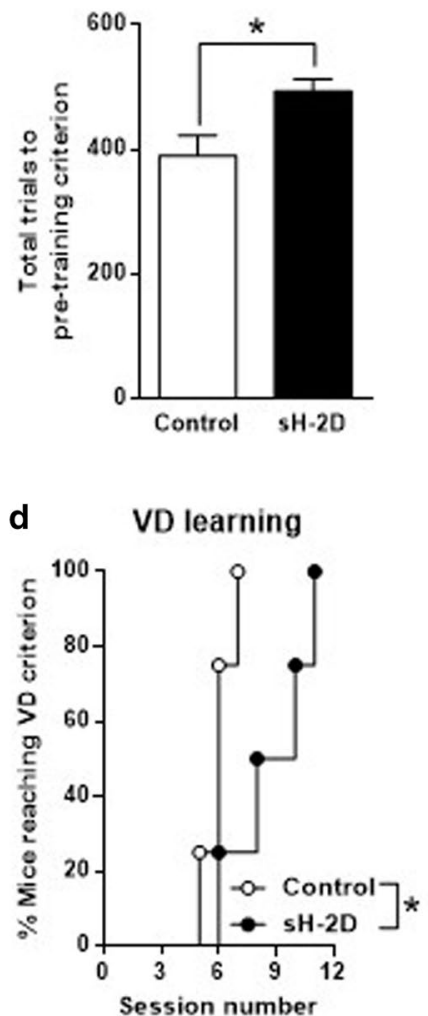

g Total normal trials

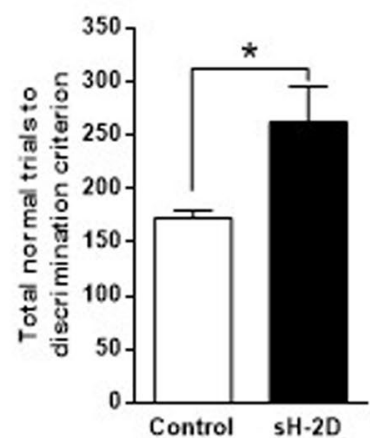

e

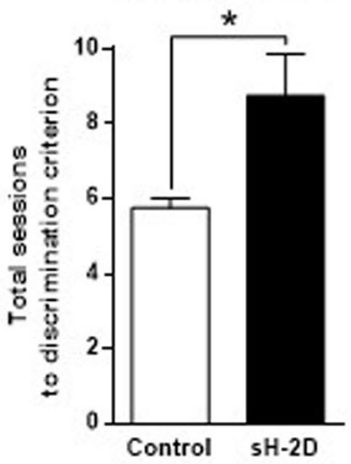

h Total correction trials

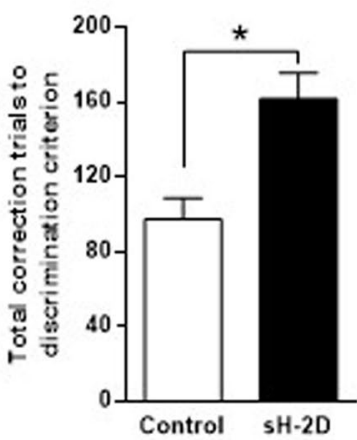

Fig. 2 Performance of $\mathrm{sH}-2 \mathrm{D}$-expressing mice in pretraining and VD task. a Introduction of incorrect response in the pretraining. $\mathbf{b}$ Total number of trials to reach the criterion in the pretraining session. $\mathbf{c}$ In the VD task, two stimuli (fan and marble) were presented simultaneously. The fan was shown as the correct response associated with a reward and the marble was an incorrect response with no reward. $\mathbf{d} V D$ learning. e Total number of sessions, $\mathbf{f}$ trials, $\mathbf{g}$ normal trials, and $\mathbf{h}$ correction trials to reach the discrimination criterion. Values indicate the mean $\pm S E M$ [control $(n=4$ mice) and $\mathrm{sH}-2 \mathrm{D}(\mathrm{n}=4$ mice) $]$

in sH-2D-expressing mice showed significantly fewer intersections (group, $\mathrm{F}(1,22)=26.71, \mathrm{P}<0.0001$; intersection, $\mathrm{F}(5,110)=17.57, \mathrm{P}<0.0001$; group $\times$ intersection, $\mathrm{F}(5,110)=1.543, \mathrm{P}=0.1824$; Fig. 4g), shorter lengths (group, $\mathrm{F}(1,22)=32.42, \mathrm{P}<0.0001$; length, $\mathrm{F}(5$, $110)=22.38,<0.0001$; group $\times$ length, $F(5,110)=2.457$,
$\mathrm{P}=0.0376 ;$ Fig. 4h), and less nodes (group, $\mathrm{F}(1$, $22)=9.79, \mathrm{P}<0.0001$; node, $\mathrm{F}(5,110)=12.63, \mathrm{P}<0.01$; group $\times$ node, $\mathrm{F}(5,110)=1.54, \mathrm{P}=0.1844 ;$ Fig. $4 \mathrm{i})$ and endings (group, $\mathrm{F}(1,22)=15.99, \mathrm{P}=0.0006$; ending, $F(5,110)=10.48,<0.0001$; group $\times$ ending, $F(5$, $110)=2.14, P=0.0664$; Fig. $4 \mathrm{j}$ ) per Sholl segment than 


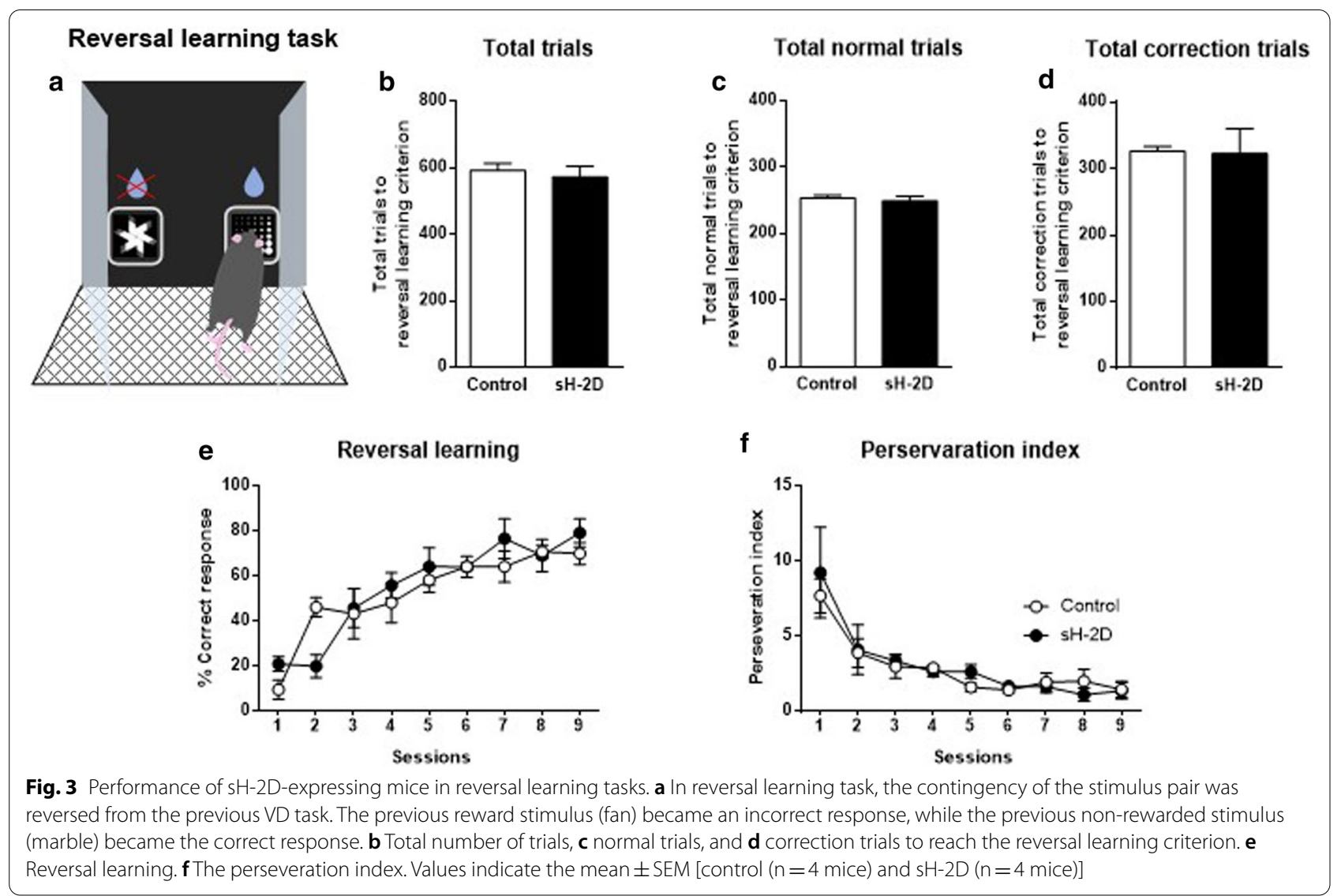

those in control mice. These results suggest that overexpression of astroglial MHCI in the MPFC decreases the dendritic complexity in the striatum.

\section{Dendritic spine formation in the DMS and DLS of $\mathrm{sH}-2 \mathrm{D}$-expressing mice}

Because the dendritic complexity is decreased in the striatum of $\mathrm{sH}-2 \mathrm{D}$-expressing mice, we hypothesized the overexpression of astroglial MHCI in the mPFC may disrupted the spine maturation process. Therefore, we further analyzed the density of spine and its subtypes (Fig. 5a). The number of spines was significantly decreased in the DMS $(\mathrm{t}(22)=3.42, \mathrm{P}=0.0025$; Fig. $5 \mathrm{~b})$ and DLS $(\mathrm{t}(22)=3.64, \mathrm{P}=0.0028$; Fig. $5 \mathrm{c})$ of sH-2D-expressing mice as compared to those in control mice. Figure $5 \mathrm{~d}$ shows the criterion to determine the spine subtypes. The thin and stubby types of spine were significantly decreased in both DMS (thin, $\mathrm{t}(22)=2.51$, $\mathrm{P}=0.0199$; stubby, $\mathrm{t}(22)=3.70, \mathrm{P}=0.0012$; Fig. $5 \mathrm{e})$ and DLS (thin, $\mathrm{t}(22)=2.42, \mathrm{P}=0.0238$; stubby, $\mathrm{t}(22)=3.25$, $\mathrm{P}=0.0037$; Fig. 5f). These results suggest that overexpression of astroglial MHCI in the MPFC decreases the thin and stubby types of spine in the striatum.

\section{Discussion}

Touchscreen-based behavioral assays are parallel with computerized tasks used in human patients [16, 31]. Mice carrying human disease-related genetic mutations exhibit cognitive impairments in the touchscreen-based VD task [17-20, 32]. Previous studies suggested that normal performance in the VD task depends on the intact function of the corticostriatal circuit $[18,21]$, which consists of the PFC, striatum, and thalamus, and is considered to be important for learning behaviors in humans, primates, and rodents [33-35]. Therefore, the function of the mPFC was tested using the VD task, which relies on this area and its projection terminals on the computation of cognitive outputs with high translational validity. The first novel result of the present study is regional and cell specificities in the role of astroglial MHCI in the touchscreen-based VD task. The animal model used here allowed astrocytes to be specifically targeted within the mPFC, without affecting other types of cells, by using the AAV under the control of the GfaABC1D promotor [15].

The task started with 5 stages of pretraining prior to the VD task. By gradually completing the 5 stages, mice learned how to operate the touchscreen to get a reward. The results obtained showed that the performance of 


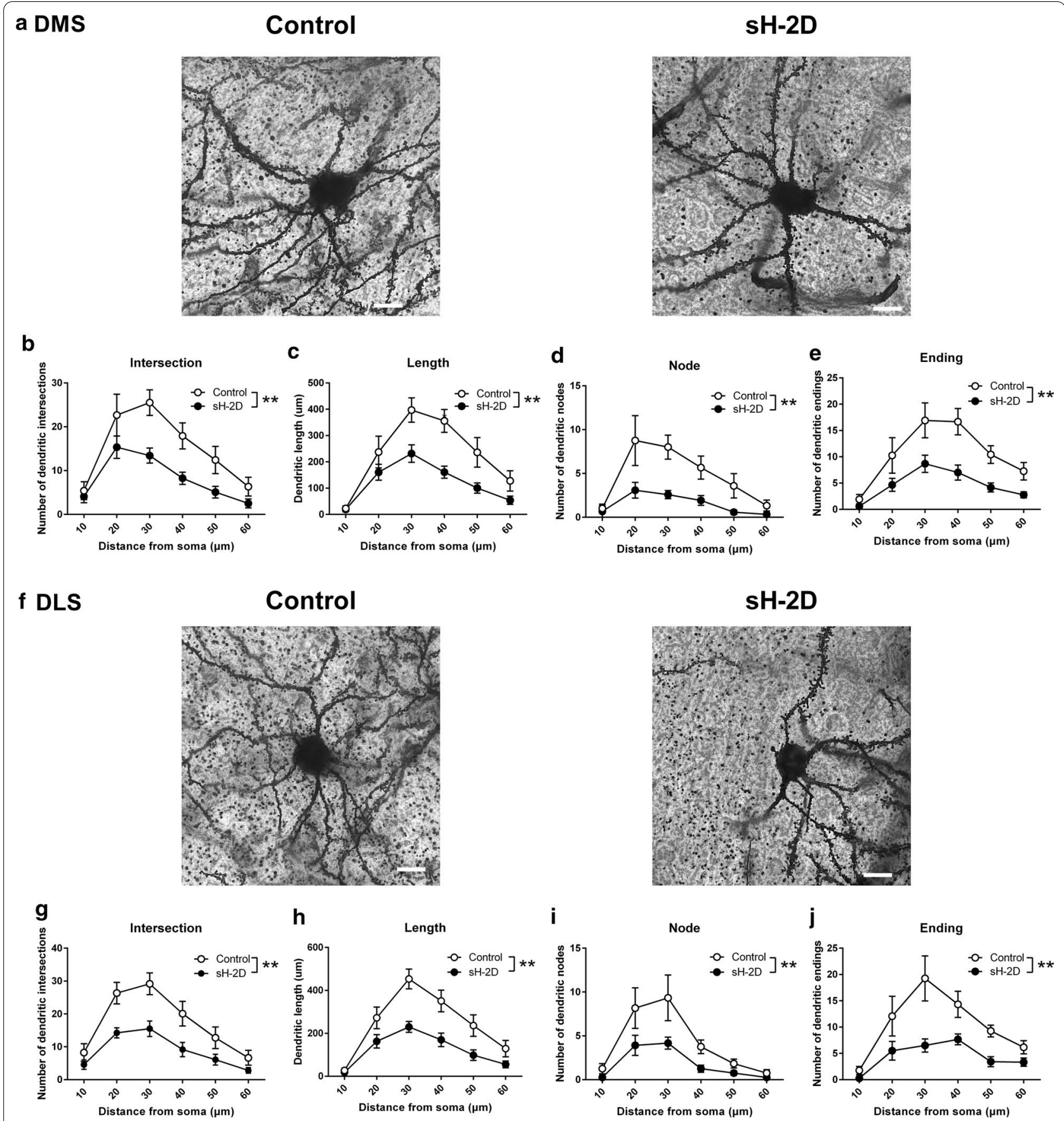

Fig. 4 Morphology of DMS and DLS neurons in SH-2D-expressing mice. a Representative images show DMS neurons in control and sH-2D-expressing mice. $\mathbf{b}$ Quantification of the intersection, $\mathbf{c}$ length, $\mathbf{d}$ node, and $\mathbf{e}$ ending of DMS neurons in control and sH-2D-expressing mice. f Representative images showing DLS neurons in control and sH-2D-expressing mice. $\mathbf{g}$ Quantification of the intersection, $\mathbf{h}$ length, $\mathbf{i}$ node, and $\mathbf{j}$ ending of DMS neurons in control and $\mathrm{SH}-2 \mathrm{D}$-expressing mice. Scale bar indicates $10 \mu \mathrm{m}$. ${ }^{* *} \mathrm{p}<0.01$ significantly different from control mice. DMS, dorsomedial striatum; DLS, dorsolateral striatum. Values indicate the mean \pm SEM [Control ( $n=12$ neurons from 4 mice) and sH-2D ( $n=12$ neurons from 4 mice)]

sH-2D-expressing mice was normal in the response phase sessions (stages 1-4), but impaired in the punish phase session (stage 5). Therefore, sH-2D-expressing mice appear to have normal visuospatial and motor functions, but impaired reward-associated discriminative learning. Similar to stage 5, the VD task requires to 


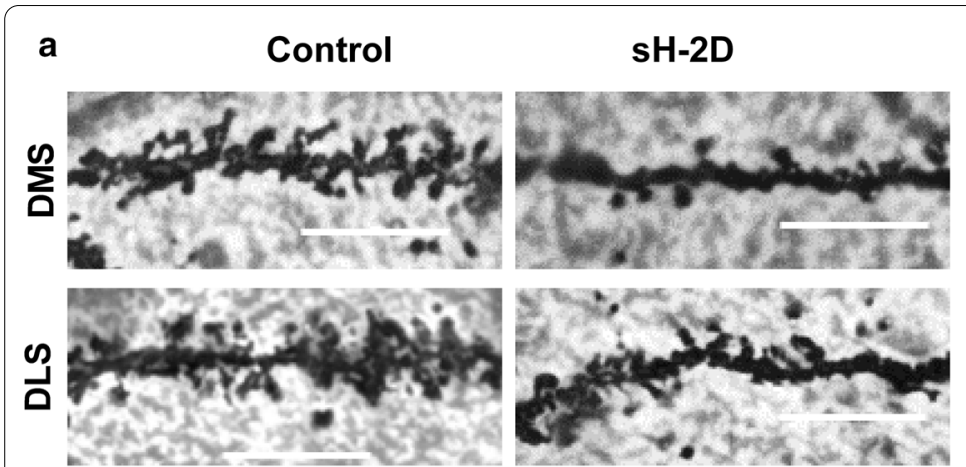

d Filopodia



e

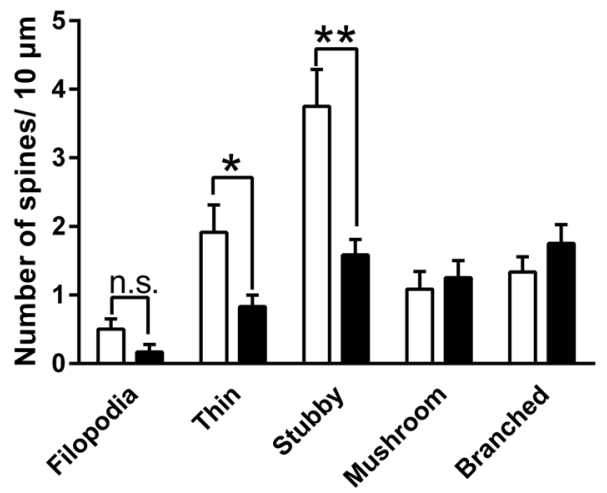

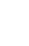

DMS

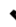

b

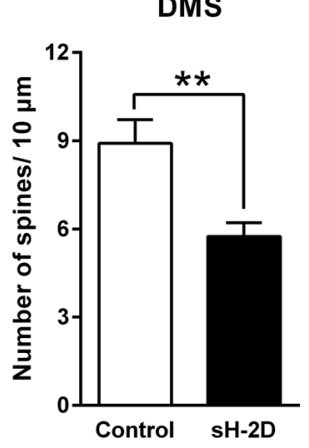

c

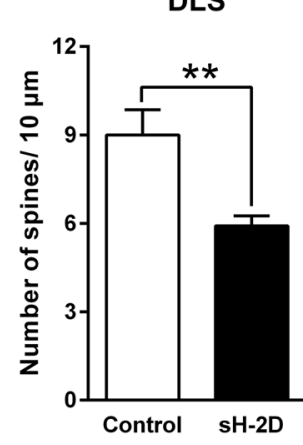
(1)

f

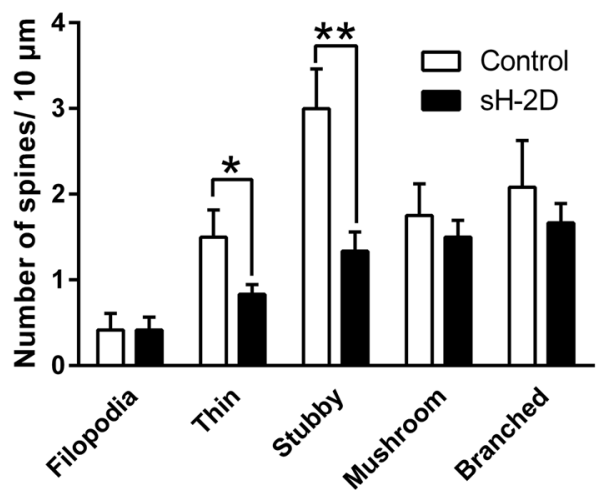

Fig. 5 Dendritic spine formation in the DMS and DLS in sH-2D-expressing mice. a Representative images show DMS and DLS spines in control and sH-2D-expressing mice. Quantification of the spines in the $\mathbf{b}$ DMS and $\mathbf{c}$ DLS. $\mathbf{d}$ Morphological classification of dendritic spines. Quantification of the filopodia, thin, stubby, mushroom and branched spines in the e DMS and $\mathbf{f}$ DLS. ${ }^{*} p<0.05$; ${ }^{* *} p<0.01$ significantly different from control mice. DMS, dorsomedial striatum; DLS, dorsolateral striatum. Scale bar indicates $10 \mu \mathrm{m}$. Values indicate the mean \pm SEM [control ( $\mathrm{n}=12$ dendrites from 4 mice) and $\mathrm{sH}-2 \mathrm{D}(\mathrm{n}=12$ dendrites from 4 mice)]

learn that one of two stimuli (marble and fan) simultaneously displayed on the screen is associated with the reward. The total numbers of sessions, trials, normal trials, and correction trials were significantly higher in $\mathrm{sH}-2 \mathrm{D}$-expressing mice than in control mice, indicating that reward learning was significantly impaired by the overexpression of MHCI in astrocytes in the mPFC. However, no significant differences were observed between sH-2D-expressing mice and control mice in the performance of reversal learning, in which the previously incorrect stimulus becomes the correct stimulus and vice versa. The perseveration index, a paradigm that is often used in reversal learning to evaluate behavioral flexibility in mice, was also similar between two groups of mice. Previous studies have indicated that activation of astrocytes in the MPFC impairs attention and reversal learning functions $[36,37]$, and lesions in the MPFC have a negative impact on the performance of reversal learning [38]. These previous findings suggest a critical role for the mPFC in reversal learning. The normal capability for reversal learning in $\mathrm{sH}-2 \mathrm{D}$-expressing mice indicates that behavioral flexibility was minimally affected 
by the overexpression of astroglial MHCI in the mPFC. One might concern the type I error because the number of mice used in the touchscreen-based tasks is quite low. We cannot exclude the possibility, but it should be noted that this operant behavioral test is an automated one with high reproducibility and low variability, which is more sensitive to detect cognitive abnormality in mice than the other respondent behavioral tests such as the novel object recognition test [16-18, 39].

Astrocytes play critical roles in CNS homeostasis by supporting neuronal metabolism and excitability, structuring the blood-brain-barrier, and limiting the synapse microenvironment [40]. They provide neurotrophic support, promote synapse formation and plasticity, and regulate synaptic transmission by interacting with dendritic spines and neuronal cell bodies [41-43]. Combining our previous findings [15] with the VD deficit observed here, it is suggested that the astroglial MHCI may affect neighboring cells and lead to a reduction in dendritic spine density with microglial activation and then decrease in parvalbumin-positive interneurons. These morphological changes may result in dysfunctions in the $\mathrm{MPFC}$ associated with cognitive function including novel object recognition and VD learning [15, 44].

Corticostriatal projections are massive and broad and arise from all cortical regions $[45,46]$. Pyramidal neurons in the $\mathrm{mPFC}$ provide cortical input, and their axons terminate primarily on the spines of striatal medium spiny neurons. These medium spiny neurons represent more than $90 \%$ of the striatal neuronal population in rodents [45]. Instrumental action and outcome behaviors depend on the striatum and its connections with the mPFC [22, 23]. Neuronal manipulations or lesion studies demonstrated that the dorsal striatum is associated with VD learning $[18,21]$. Cortical synaptic inputs into the striatum are important for the maturation of the dendritic arborization of striatal medium spine neurons [47]. The present study has raised an important question if the neuronal changes could be observed only in the areas directly linked to the mPFC.

We have previously demonstrated that treatment with GW4869 which impairs exosome synthesis significantly ameliorates the behavioral and neuropathological changes in sH-2D-expressing mice [15]. Accordingly, the overexpression of MHCI in astrocytes of mPFC induces microglial proliferation and the decrease in neuronal numbers of the mPFC, and affects dendritic complexity and spine density in the striatum, specifically the lowered number of spinal thin and stubby types. It is possible that all of these could be triggered by the exosomes that are secreted by MHCI-expressing astrocytes in the mPFC. The manipulation in the mPFC may have delayed the spine maturation thereafter leading to the decreased dendritic complexity in the striatum. More direct evidence is needed to test this hypothesis in future studies.

We previously demonstrated that treatment with polyinosinic-polycytidylic acid (poly:C) in adult mice significantly increased $\mathrm{MCHI}$, interferon, tumor necrosis factor-a, and interleukin- 6 mRNA expression levels in the mPFC [15]. Under pathological conditions, such as viral infection, astrocytes secrete several inflammatory cytokines and chemokines that interrupt local immune responses, which may contribute to the expansion of primary lesions, leading to further neuronal loss [48, 49]. Activated microglia induce neuronal degeneration or death, both of which are associated with mental disorders such as schizophrenia and depression [5052]. Overexpression of astroglial MHCI in the mPFC increases the number of microglia and the glutamatergic transporter expression (e.g. glutamate transporter 1) $[15,53]$. We speculate the expression of striatal microglia may have altered by the glutamatergic projection from mPFC. As mentioned above, the inflammatory cytokines and chemokines may contribute to these processes, but future studies are necessary to prove it.

In conclusion, the present results suggest that the overexpression of MHCI in astrocytes in the mPFC results in impaired VD learning, which is associated with decreased dendritic complexity and spine density in medium spine neurons in the dorsal striatum and mPFC.

\section{Abbreviations \\ AAV: Adeno-associated virus; CNS: Central nervous system; DLS: Dorsolateral striatum; DMS: Dorsomedial striatum; GFAP: Glial fibrillary acidic protein; ITI: Inter-trial interval; MHC: Major histocompatibility complex; MHCI: MHC class l; PCR: Polymerase chain reaction; VD: Visual discrimination; mPFC: Medial prefrontal cortex; poly:C: Polyinosinic-polycytidylic acid; SH-2D: Soluble form of $\mathrm{H}-2 \mathrm{D} ; \mathrm{s} \mathrm{H}-2 \mathrm{D}$-expressing mice: Mice expressing the soluble form of $\mathrm{H}-2 \mathrm{D}$ in the $\mathrm{mPFC}$}

\section{Acknowledgements}

B.W. thanks the support by the Ajinomoto Scholarship Foundation and the Otsuka Toshimi Scholarship Foundation.

\section{Authors' contributions}

$\mathrm{BW}$ and $\mathrm{KH}$ devised the project and the main conceptual ideas, participated in all experiments and drafted the manuscript. AS and NI assisted with experiments. ToN contributed to the manuscript discussion. TaN and KY supervised the work and finalized the manuscript. All authors read and approved the final manuscript.

\section{Funding}

This work was supported by the following funding sources: KAKENHI Grant Numbers JP17H01380, JP17H02220, JP17H04031, JP17H04252 and 2OH03428, from Japan Society for the Promotion of Science, JP20dm0107087 from Japan Agency for Medical Research and Development, and from The Uehara Memorial Foundation.

Availability of data and materials

All data used in this study are available from the corresponding author on reasonable request. 


\section{Ethics approval and consent to participate}

Animals were handled in accordance with the guidelines established by the Institutional Animal Care and Use Committee of Nagoya University, the Guiding Principles for the Care and Use of Laboratory Animals approved by the Japanese Pharmacological Society, and the National Institutes of Health Guide for the Care and Use of Laboratory Animals.

\section{Consent for publication}

Not applicable.

\section{Competing interests}

None of the authors have any conflicts of interests related to this work.

\section{Author details}

${ }^{1}$ Department of Neuropsychopharmacology and Hospital Pharmacy, Nagoya University Graduate School of Medicine, 65 Tsurumai-cho, Showa-ku, Nagoya 466-8560, Japan. ${ }^{2}$ Advanced Diagnostic System Research Laboratory, Fujita Health University Graduate School of Health Sciences, Toyoake 470-1192, Japan.

Received: 17 August 2020 Accepted: 2 December 2020

Published online: 14 December 2020

\section{References}

1. Murphy JB, Sturm E. Conditions determining the transplantability of tissues in the brain. J Exp Med. 1923;38(2):183-97.

2. Joly E, Mucke L, Oldstone MB. Viral persistence in neurons explained by lack of major histocompatibility class I expression. Science. 1991:253(5025):1283-5.

3. Garay PA, McAllister AK. Novel roles for immune molecules in neural development: implications for neurodevelopmental disorders. Front Synaptic Neurosci. 2010;2:136.

4. Stevens B, Allen NJ, Vazquez LE, Howell GR, Christopherson KS, Nouri N, et al. The classical complement cascade mediates CNS synapse elimination. Cell. 2007;131(6):1164-78.

5. Shatz CJ. MHC class I: an unexpected role in neuronal plasticity. Neuron. 2009:64(1):40-5.

6. Heemels MT, Ploegh H. Generation, translocation, and presentation of MHC class I-restricted peptides. Annu Rev Biochem. 1995;64:463-91.

7. Natarajan K, Li H, Mariuzza RA, Margulies DH. MHC class I molecules, structure and function. Rev Immunogenet. 1999;1 (1):32-46.

8. Boulanger LM. MHC class I in activity-dependent structural and functional plasticity. Neuron Glia Biol. 2004;1(3):283-9.

9. Elmer BM, Estes ML, Barrow SL, MCAllister AK. MHCl requires MEF2 transcription factors to negatively regulate synapse density during development and in disease. J Neurosci. 2013;33(34):13791-804.

10. Lee H, Brott BK, Kirkby LA, Adelson JD, Cheng S, Feller MB, et al. Synapse elimination and learning rules co-regulated by $\mathrm{MHC}$ class I H2-Db. Nature. 2014;509(7499):195-200.

11. Biesmans S, Meert TF, Bouwknecht JA, Acton PD, Davoodi N, De Haes P, et al. Systemic immune activation leads to neuroinflammation and sickness behavior in mice. Mediat Inflamm. 2013;2013:271359.

12. Norden DM, Trojanowski PJ, Walker FR, Godbout JP. Insensitivity of astrocytes to interleukin 10 signaling following peripheral immune challenge results in prolonged microglial activation in the aged brain. Neurobiol Aging. 2016:44:22-41.

13. Foster JA, Quan N, Stern EL, Kristensson K, Herkenham M. Induced neuronal expression of class I major histocompatibility complex mRNA in acute and chronic inflammation models. J Neuroimmunol. 2002;131(1-2):83-91.

14. Ng YK, Ling EA. Induction of major histocompatibility class II antigen on microglial cells in postnatal and adult rats following intraperitoneal injections of lipopolysaccharide. Neurosci Res. 1997;28(2):111-8.

15. Sobue A, Ito N, Nagai T, Shan W, Hada K, Nakajima A, et al. Astroglial major histocompatibility complex class I following immune activation leads to behavioral and neuropathological changes. Glia. 2018;66(5):1034-52.

16. Bussey TJ, Holmes A, Lyon L, Mar AC, McAllister KA, Nithianantharajah $J$, et al. New translational assays for preclinical modelling of cognition in schizophrenia: the touchscreen testing method for mice and rats. Neuropharmacology. 2012;62(3):1191-203.

17. Nithianantharajah J, McKechanie AG, Stewart TJ, Johnstone M, Blackwood $\mathrm{DH}$, St Clair D, et al. Bridging the translational divide: identical cognitive touchscreen testing in mice and humans carrying mutations in a diseaserelevant homologous gene. Sci Rep. 2015;5:14613.

18. Wulaer B, Nagai T, Sobue A, Itoh N, Kuroda K, Kaibuchi K, et al. Repetitive and compulsive-like behaviors lead to cognitive dysfunction in Disc1(Delta2-3/Delta2-3) mice. Genes Brain Behav. 2018;17(8):e12478.

19. Nithianantharajah J, Komiyama NH, McKechanie A, Johnstone M, Blackwood DH, St Clair D, et al. Synaptic scaffold evolution generated components of vertebrate cognitive complexity. Nat Neurosci. 2013;16(1):16-24.

20. Saito R, Koebis M, Nagai T, Shimizu K, Liao J, Wulaer B, et al. Comprehensive analysis of a novel mouse model of the 22q11.2 deletion syndrome: a model with the most common 3.0-Mb deletion at the human $22 \mathrm{q} 11.2$ locus. Transl Psychiatry. 2020;10(1):35.

21. Brigman JL, Daut RA, Wright T, Gunduz-Cinar O, Graybeal C, Davis MI, et al. GluN2B in corticostriatal circuits governs choice learning and choice shifting. Nat Neurosci. 2013;16(8):1101-10.

22. Corbit LH, Nie H, Janak PH. Habitual alcohol seeking: time course and the contribution of subregions of the dorsal striatum. Biol Psychiatry. 2012;72(5):389-95.

23. Murray JE, Belin D, Everitt BJ. Double dissociation of the dorsomedial and dorsolateral striatal control over the acquisition and performance of cocaine seeking. Neuropsychopharmacology. 2012;37(11):2456-66.

24. Cox J, Witten IB. Striatal circuits for reward learning and decision-making. Nat Rev Neurosci. 2019;20(8):482-94.

25. Meziane H, Ouagazzal AM, Aubert L, Wietrzych M, Krezel W. Estrous cycle effects on behavior of $\mathrm{C} 57 \mathrm{BL} / 6 \mathrm{~J}$ and BALB/CByJ female mice: implications for phenotyping strategies. Genes Brain Behav. 2007;6(2):192-200.

26. Wulaer B, Kunisawa K, Hada K, Suento WJ, Kubota H, lida T, et al. Shati/ Nat8l deficiency disrupts adult neurogenesis and causes attentional impairment through dopaminergic neuronal dysfunction in the dentate gyrus. J Neurochem. 2020. https://doi.org/10.1111/jnc.15022.

27. Nagai T, Nakamuta S, Kuroda K, Nakauchi S, Nishioka T, Takano T, et al. Phosphoproteomics of the dopamine pathway enables discovery of Rap1 activation as a reward signal in vivo. Neuron. 2016;89(3):550-65.

28. Paxinos G, Franklin KBJ. The mouse brain in stereotaxic coordinates. Amsterdan: Elsevier Academic Press; 2004.

29. Horner AE, Heath CJ, Hvoslef-Eide M, Kent BA, Kim CH, Nilsson SR, et al. The touchscreen operant platform for testing learning and memory in rats and mice. Nat Protoc. 2013;8(10):1961-84

30. Smith Y, Surmeier DJ, Redgrave P, Kimura M. Thalamic contributions to basal ganglia-related behavioral switching and reinforcement. J Neurosci. 2011;31(45):16102-6

31. Hvoslef-Eide M, Mar AC, Nilsson SR, Alsio J, Heath CJ, Saksida LM, et al. The NEWMEDS rodent touchscreen test battery for cognition relevant to schizophrenia. Psychopharmacology. 2015;232(21-22):3853-72.

32. Yang M, Lewis FC, Sarvi MS, Foley GM, Crawley JN. 16p11.2 Deletion mice display cognitive deficits in touchscreen learning and novelty recognition tasks. Learn Mem. 2015;22(12):622-32.

33. Haber SN. The place of dopamine in the cortico-basal ganglia circuit. Neuroscience $2014 \cdot 282 \cdot 248-57$.

34. Gunaydin LA, Kreitzer AC. Cortico-basal ganglia circuit function in psychiatric disease. Annu Rev Physiol. 2016;78:327-50.

35. Ng TH, Alloy LB, Smith DV. Meta-analysis of reward processing in major depressive disorder reveals distinct abnormalities within the reward circuit. Transl Psychiatry. 2019;9(1):293.

36. Birrell JM, Brown VJ. Medial frontal cortex mediates perceptual attentional set shifting in the rat. J Neurosci. 2000;20(11):4320-4.

37. Nagai J, Rajbhandari AK, Gangwani MR, Hachisuka A, Coppola G, Masmanidis SC, et al. Hyperactivity with disrupted attention by activation of an astrocyte synaptogenic cue. Cell. 2019;177(5):1280-92 e20.

38. Latif-Hernandez A, Shah D, Ahmed T, Lo AC, Callaerts-Vegh Z, Van der Linden $A$, et al. Quinolinic acid injection in mouse medial prefrontal cortex affects reversal learning abilities, cortical connectivity and hippocampal synaptic plasticity. Sci Rep. 2016;6:36489.

39. Kuroda K, Yamada S, Tanaka M, lizuka M, Yano H, Mori D, et al. Behavioral alterations associated with targeted disruption of exons 2 and 3 of the Disc1 gene in the mouse. Hum Mol Genet. 2011;20(23):4666-83. 
40. Colombo E, Farina C. Astrocytes: key regulators of neuroinflammation. Trends Immunol. 2016;37(9):608-20.

41. Ben Achour S, Pascual O. Astrocyte-neuron communication: functional consequences. Neurochem Res. 2012;37(11):2464-73.

42. Freeman MR, Rowitch DH. Evolving concepts of gliogenesis: a look way back and ahead to the next 25 years. Neuron. 2013;80(3):613-23.

43. Haber M, Zhou L, Murai KK. Cooperative astrocyte and dendritic spine dynamics at hippocampal excitatory synapses. J Neurosci. 2006:26(35):8881-91.

44. Cebrian C, Zucca FA, Mauri P, Steinbeck JA, Studer L, Scherzer CR, et al. MHC-I expression renders catecholaminergic neurons susceptible to T-cell-mediated degeneration. Nat Commun. 2014;5:3633.

45. Bolam JP, Hanley JJ, Booth PA, Bevan MD. Synaptic organisation of the basal ganglia. J Anat. 2000;196(Pt 4):527-42.

46. Albin RL, Young AB, Penney JB. The functional anatomy of basal ganglia disorders. Trends Neurosci. 1989;12(10):366-75.

47. Buren C, Tu G, Parsons MP, Sepers MD, Raymond LA. Influence of cortical synaptic input on striatal neuronal dendritic arborization and sensitivity to excitotoxicity in corticostriatal coculture. J Neurophysiol. 2016:116(2):380-90.
48. Xie L, Yang SH. Interaction of astrocytes and T cells in physiological and pathological conditions. Brain Res. 2015;1623:63-73.

49. Becher B, Spath S, Goverman J. Cytokine networks in neuroinflammation. Nat Rev Immunol. 2017;17(1):49-59.

50. Monji A, Kato TA, Mizoguchi Y, Horikawa H, Seki Y, Kasai M, et al. Neuroinflammation in schizophrenia especially focused on the role of microglia. Prog Neuropsychopharmacol Biol Psychiatry. 2013;42:115-21.

51. Zhu X, Levasseur PR, Michaelis KA, Burfeind KG, Marks DL. A distinct brain pathway links viral RNA exposure to sickness behavior. Sci Rep. 2016;6:29885

52. Hong H, Kim BS, Im HI. Pathophysiological role of neuroinflammation in neurodegenerative diseases and psychiatric disorders. Int Neurourol J. 2016;20(Suppl 1):S2-7.

53. Ong WY, Stohler CS, Herr DR. Role of the prefrontal cortex in pain processing. Mol Neurobiol. 2019;56(2):1137-66.

\section{Publisher's Note}

Springer Nature remains neutral with regard to jurisdictional claims in published maps and institutional affiliations.
Ready to submit your research? Choose BMC and benefit from:

- fast, convenient online submission

- thorough peer review by experienced researchers in your field

- rapid publication on acceptance

- support for research data, including large and complex data types

- gold Open Access which fosters wider collaboration and increased citations

- maximum visibility for your research: over $100 \mathrm{M}$ website views per year

At BMC, research is always in progress.

Learn more biomedcentral.com/submissions 